\title{
A new approach in Presurgical Infant Orthopedics using an Active Alveolar Molding Appliance in the management of bilateral cleft lip and palate patient: A Case Report
}

\author{
Dr. N. Retnakumari. MDS, M.Phil, Dr. Manuja Vargheese, Dr. Madhu .S, \\ Dr. Divya. S
}

\begin{abstract}
Cleft lip and palate can arise with considerable variation in severity and form. Generally, the wider, more extensive clefts are associated with more significant nasolabial deformity. These clefts, deficient in hard and soft tissue elements, present a significant surgical challenge to achieve a functional and cosmetic outcome. A finer scar forms when a surgical incision heals under less rather than more tension. The principal objective of presurgical nasoalveolar molding (NAM) is to reduce the severity of the initial cleft deformity. The nasoalveolar molding appliance is a modern presurgical orthopedic device that allows for a positive growth of the alveolar ridges into an improved arch form as well as reshaping of the flattened nose into a more symmetrical shape. As a result of the presurgical appliance, the nose and lip are allowed to heal under minimal tension, thereby reducing scar formation and improving the esthetic result. This case report describes the management of a bilateral cleft lip and palate case with the use of an active alveolar molding appliance which retracts the forwardly placed premaxilla into a more esthetic position prior to the primary surgical repair. Keywords: Nasoalveolar Molding, Presurgical Infant Orthopedics, Active Alveolar Molding Appliance, Cleft Lip And Cleft Palate
\end{abstract}

\section{Introduction:}

Advances in cleft lip and palate reconstructive surgery have led to improved quality of repair. However, difficulties still remain in preventing deficiencies, scarring, and asymmetry of the maxillofacial hard and soft tissues. It has become apparent that addressing unilateral and the even more challenging bilateral cleft lip and palate anomalies requires an interdisciplinary effort to restore the nasolabial complex to approximately normal anatomy. The use of pre-surgical infant orthopedic appliances, or molding therapy, has aided significantly in reducing the size of clefts, while actively repositioning the surrounding soft tissues including the prolabium, nasal dome and alar cartilages. If there is close coordination of the pre-surgical phase of treatment and the surgical method employed, these new techniques can greatly improve upon facial esthetics and minimize the extent of the surgery and the overall number of surgical procedures. ${ }^{1,2}$

Grayson and Cutting were one of the first to combine the concepts of presurgical orthopedics and preoperative treatment of the cleft lip nasal deformity. They developed the concept of nasoalveolar molding, which combined a nasal molding stent with a passive, presurgical molding appliance in treating cleft lip and palate infants. $^{3}$

In presurgical orthopedic therapy, active and passive appliances are used to mold the maxillary, alveolar, and nasal tissues of an infant with a unilateral or bilateral cleft lip and palate Active appliances are fixed intraorally and apply traction through mechanical means such as elastic chains, screws, and plates. Passive appliances maintain the distance between the 2 maxillary segments while external force is applied primarily to reposition the segments posteriorly. External force can be applied by external taping of the lip, a head cap with elastic straps across the prolabium, or a surgical lip adhesion.

Active maxillary appliances move alveolar cleft segments in a predetermined manner with controlled forces, whereas passive appliances deliver no force but act as a fulcrum upon which the forces created by surgical lip closure contour and mold the alveolar segments in a predictable fashion. ${ }^{4}$

A case of bilateral cleft lip and palate treated using an active alveolar molding appliance is described in this article. The active component of the appliance is the expansion screw incorporated in it which moves the premaxilla in a more desired position.

\section{Case report:}

A male child, age of 30 days was referred to the Department of Pedodontics and Preventive Dentistry, Govt. Dental College, Calicut by the Department of Pediatrics, Govt. Medical College Calicut. On clinical examination the patient had a bilateral cleft lip and palate with the forwardly positioned premaxilla. [Figure 1] The child had one thumb and two fingers in both hands, one big toe and one finger with a cleft in between them 
in both the legs. The mother also has history of cleft lip repair in childhood. The mother had one thumb and three fingers in both hands and two fingers in both legs.

An active alveolar molding appliance was decided for approximation of the alveolus and align with the premaxilla as presurgical infant orthopedic therapy. The parents were informed about the details of procedure and informed consent was obtained from them.

\section{Impression:}

\section{Method and fabrication of appliance:}

The patient was taken to the operation theatre for taking impression of the bilateral cleft in order to meet any emergency. The patient was made to lie down on the operation theatre table in supine position. The impression was taken using heavy body rubber base impression material(3M ESPE Express ${ }^{\mathrm{TM}}$ STD vinyl polysiloxane impression material putty). After removing the impression from the patient's mouth, the oral cavity was thoroughly examined for any remnant impression material. The impression was poured using dental stone and the cast was made ready for appliance fabrication.[Figure 2]

\section{Fabrication of the appliance:}

The undercuts in the cast were blocked, relief was given for frenal attachments. The acrylic plate of $2 \mathrm{~mm}$ thickness was made using self cure polymerising resin. An expansion screw (Jack screw) fully opened was incorporated into the appliance. This formed the active component of the appliance for retraction of the protruded premaxilla. All the peripheral borders were smoothened so as to avoid irritation of the mucosa. The appliance was properly trimmed and polished. The anterior component of the appliance was fabricated with two retention stops. These retention stops facilitated the attachment of elastic traps on both sides. [Figure 3]

\section{Insertion of the appliance:}

The alveolar molding appliance was inserted into the patient's mouth. The oral cavity was carefully examined for any blanching of soft tissues, whether proper relief was given for frenal attachments and if the extension of the molding plate was sufficient to serve the purpose. The elastic traps one on each side were attached to the retention button. The appliance was placed in proper position with the help of elastic straps which were adhered on to the cheeks of the child. Parents were demonstrated regarding the insertion and removal of appliance.[Figure 4]

\section{Activation of the appliance:}

The appliance was activated once in two weeks. In each visit oral cavity was examined for any ulceration or trauma due to the appliance, parents cooperation was evaluated and motivated. The appliance was activated by closing the expansion screw and relining with denture base material. [Figure 5].The activation of appliance continued till 4 months.

\section{Results:}

The premaxilla was retracted and the cleft gap was reduced with the use of this active alveolar molding appliance. This enabled better esthetic results after surgery by reducing tissue tension and scar formation.[Figure 6]

\section{Discussion:}

The alveolar molding appliance approximates the alveolar segments as close as possible before surgery and brings the premaxilla back into the position of the alveolar arch in bilateral cleft patients. When combined with primary gingivoperiosteoplasty (GPP), this potentially results in a reduced need for alveolar bone grafting during the mixed dentition period.

In addition, there are several other advantages to using a prosthetic device to place a premaxilla in a more anatomically correct position before surgical closure of the lip. First, soft tissue will be carried with the segment, leading to a decrease in the width of the defect. Second, a centrally positioned premaxillary segment provides a more ideal base for lip closure. It decreases tissue tension during the surgical procedure, and finally, it allows healed soft tissues to rest against a more normal bony anatomy. If the premaxilla is not repositioned in these extreme cases, excessive tension may develop at the surgical site, which compromises the surgical result. By using orthopedic therapy, a second operative session may be eliminated, thereby decreasing total hospitalization time and cost. ${ }^{1,3,5}$

The most common problems observed during NAM therapy are irritation to the oral mucosa, gingival tissue or nasal mucosa. Intraoral tissues may ulcerate from excessive pressure applied by the appliance. These are commonly found in the oral vestibule and on the labial side of the premaxilla. The oral and the nasal cavities of the infant should be carefully examined on each visit for ulceration and appropriate adjustments should be 
made to the moulding plate to relieve sore spots. The intranasal lining of the nasal tip can become inflamed if too much force is applied by the upper lobe of the nasal stent. The area under the horizontal prolabium band can become ulcerated if the band is too tight. ${ }^{6,7,8,9}$

Another area of tissue irritation is the cheeks. Extreme care should be taken while removing the cheek tape to avoid any irritation to the skin. Skin barrier tapes like Tegaderm ${ }^{\mathrm{TM}}$ are recommended. Slight relocation of the position of the tape during treatment is also recommended to provide rest to the tissues in case they become irritated. It is also recommended that an aloevera gel be applied to the cheeks when changing tapes. ${ }^{10}$

\section{Conclusion:}

Presurgical orthopedic devices are a controversial topic in cleft treatment. Arguments against their use include the potential for long-term growth effects and potential feeding difficulty. With increasing clinical experience, the long-term outcome and the specific role of presurgical orthopedics are becoming better defined. An active removable orthopedic appliance with reverse expansion screw could be an effective tool in alveolar molding which retracts the forwardly placed premaxilla into a more esthetic position prior to the primary surgical repair.

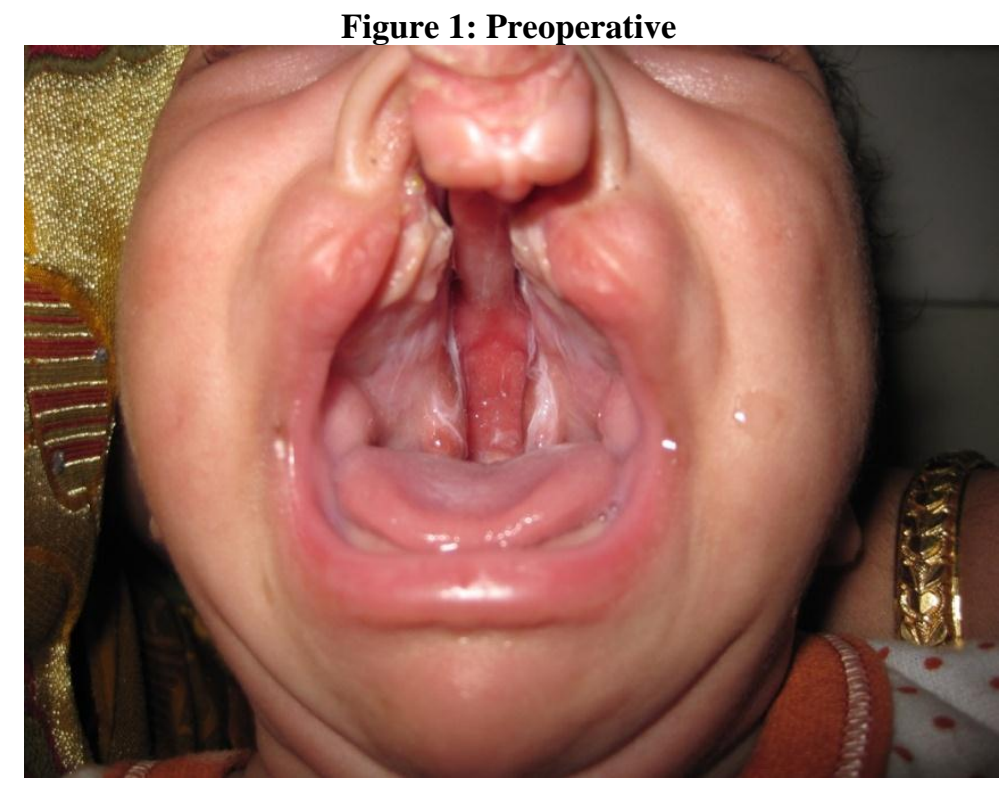

Figure 2:Heavy body silicone impression

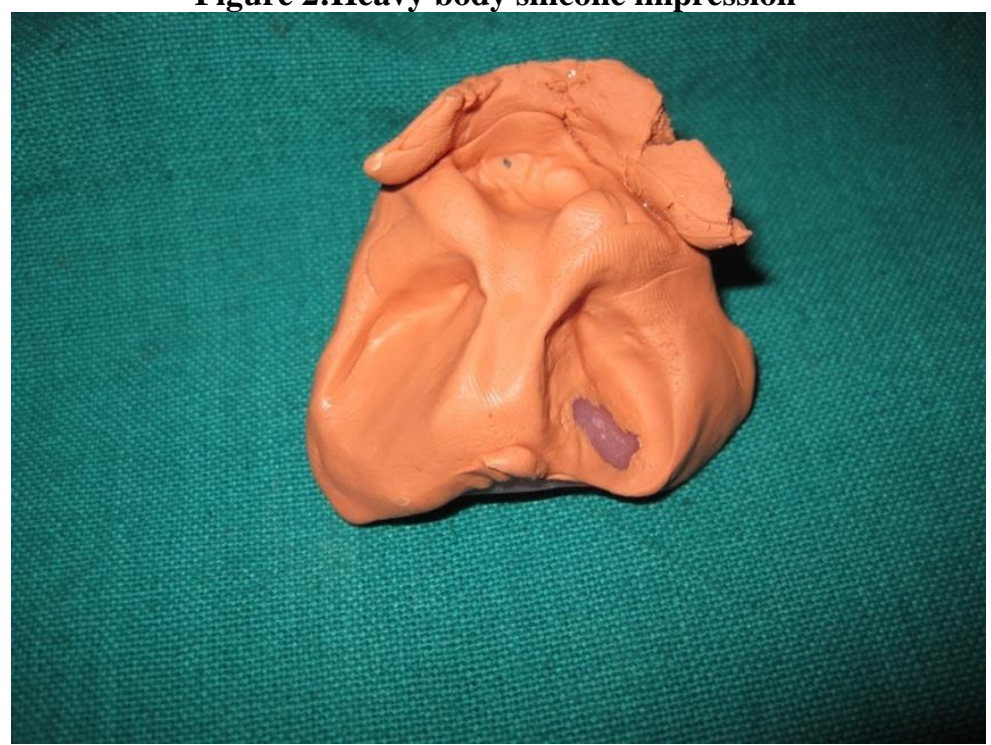


Figure 3: Active alveolar molding appliance

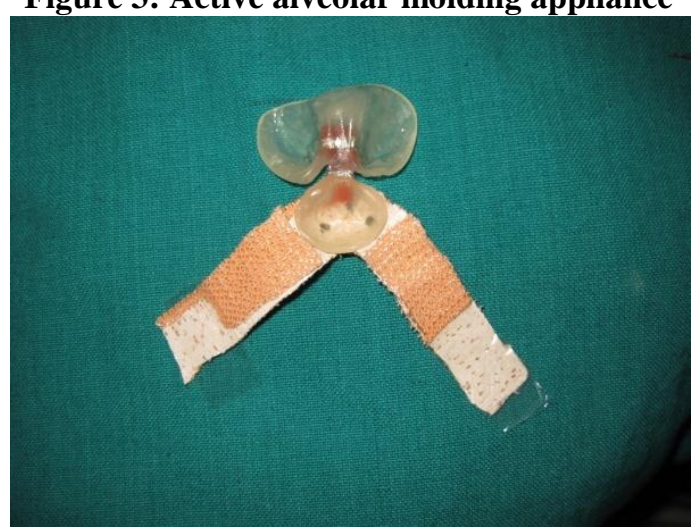

Figure 4-Molding appliance delivery \& Approximation with steri strips

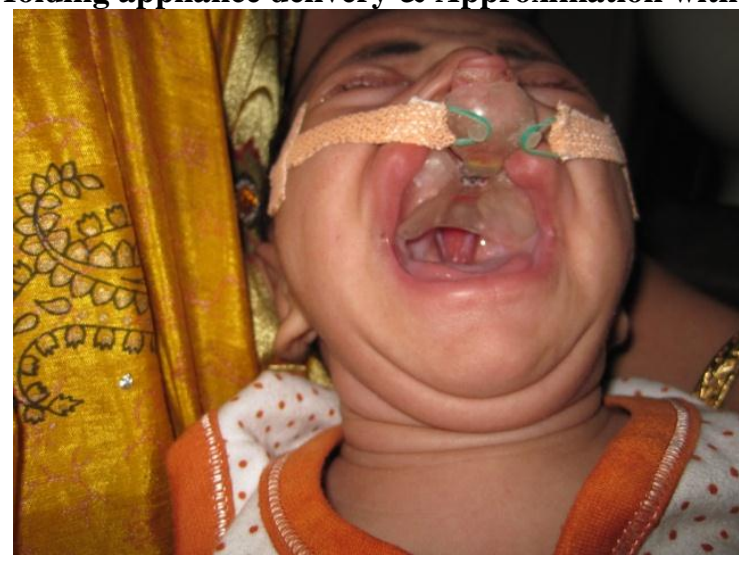

Figure 5- Activation by relining

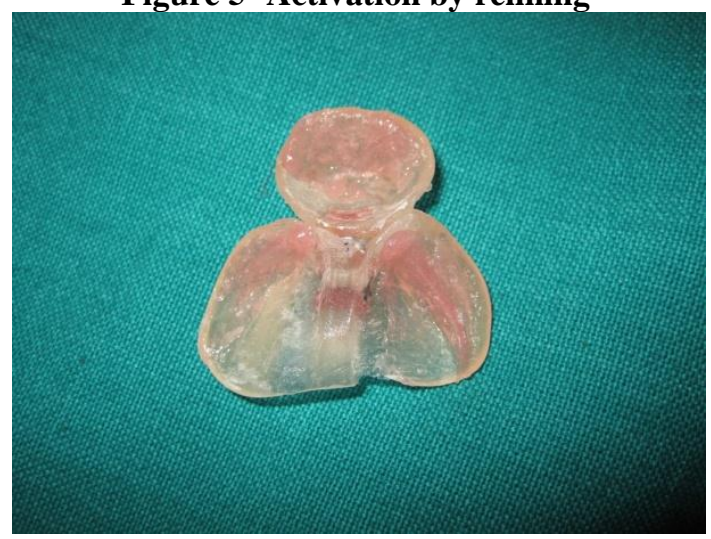

Figure 6- Post operative 


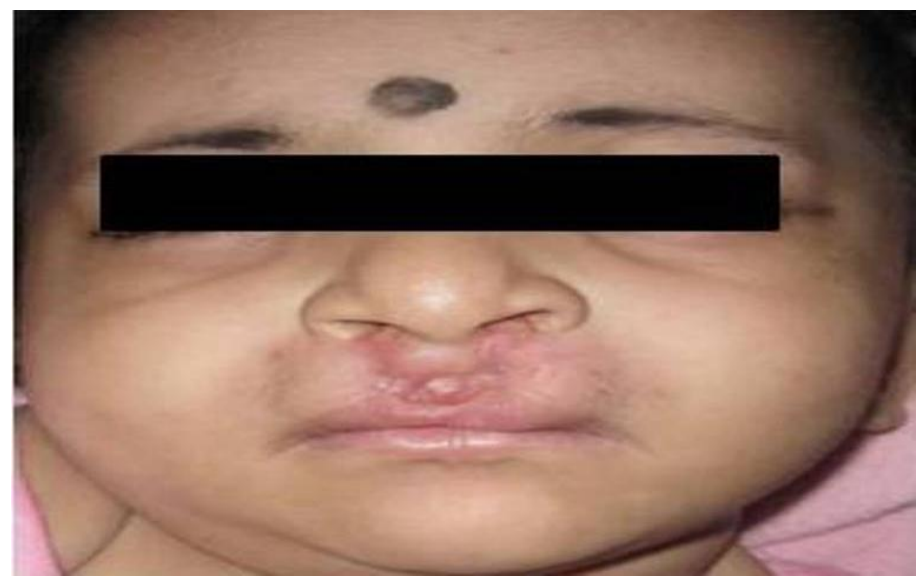

\section{References:}

[1]. Ma X, Giacona MB. Nasoalveolar Molding as Treatment for Cleft Lip and Palate: A Case Report Columbia Dental Review. 13:2024, 2009

[2]. Prasanth et al. Cleft orthopedics using Liou's technique - A Case Report, Journal of Dental Sciences and Research .2(1):122131,2011

[3]. Yang et al. Use of Nasoalveolar Molding Appliance to Direct Growth in Newborn Patient With Complete Unilateral Cleft Lip and Palate. Pediatric Dentistry. 25(3):253-256, 2003

[4]. Suri S, Tompson BD. A Modified Muscle-Activated Maxillary Orthopedic Appliance For Presurgical Nasoalveolar Molding In Infants With Unilateral Cleft Lip And Palate. Cleft palate-Craniofacial Journal. 4(3):225-229, 2004

[5]. Karimi et al. Presurgical Nasoalveolar Molding in a Neonate With Bilateral Cleft Lip and Palate: Report of a Case. J Comprehensive Pediatrics. 3(2): 86-9, 2012.

[6]. Grayson et al. Nasoalveolar Molding For Infants Born With Clefts Of The Lip, Alveolus, And Palate. Seminars In Plastic Surgery. 19(4):294-301, 2005.

[7]. Splengler et al. Presurgical Nasoalveolar Molding Therapy For The Treatment Of Bilateral Cleft Lip And Palate: A Preliminary Study, Cleft Palate-Craniofacial Journal. 43(3):321-328, 2006.

[8]. Shetty et al. Pre-Surgical Nasoalveolar Molding In Patients With Unilateral Clefts Of Lip, Alveolus And Palate - A Case Report. Annals And Essences Of Dentistry. 3(2):50-52, 2011.

[9]. Abbott et al. Nasoalveolar molding in cleft care: is it efficacious? Plast Reconstr Surg.130(3):659-66, 2012

[10]. Grayson BH, Shetye PR. Presurgical nasoalveolar moulding treatment in cleft lip and palate patients. Indian Journal of Plastic Surgery .42(3): 56-61, 2009. 